\title{
Possédez-vous un diplôme de formation continue?
}

Six ans après l'entrée en vigueur de TARMED, 17000 médecins font encore valoir 1,2 million de positions de droits acquis. Désormais, il n'est plus nécessaire de suivre une «formation continue pour prestations de droits acquis» pour pouvoir continuer à facturer ces prestations à la charge de l'assurance sociale. Il suffit d'acquérir un diplôme de formation continue d'une des $\mathbf{4 5}$ sociétés de discipline médicale reconnues.

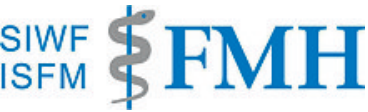

Christoph Hänggeli

Administrateur de l'Institut suisse pour la formation médicale postgraduée et continue (ISFM)
1 Hänggeli C, Kappeler O. Formation continue et droits acquis. Bulletin des médecins suisses. 2006;87(18):768-771.
Correspondance:

ISFM/FMH

Elfenstrasse 18

Postfach 170

$\mathrm{CH}-3000$ Berne 15

Tél. 0313591111

Fax 0313591112

isfm.fmh.ch

\section{Préambule}

Depuis l'entrée en vigueur de TARMED le $1^{\text {er }}$ janvier 2004, les médecins peuvent facturer une position tarifaire uniquement s'ils disposent de la «valeur intrinsèque» correspondante. Il s'agit, en l'occurrence, de la qualification professionnelle, à savoir les titres de spécialiste, les formations approfondies et les attestations de formation complémentaire réglés dans la Réglementation pour la formation postgraduée. Néanmoins, la «garantie des droits acquis» permet aux médecins de continuer d'exercer la profession comme ils le faisaient déjà avant l'introduction de TARMED et de facturer les prestations pour lesquelles ils ne possèdent pas d'attestations ou de titres.

Conformément à leur caractère juridique transitoire, les droits acquis ne se prolongent pas automatiquement. Le concept de valeur intrinsèque, normatif en la matière, exige des médecins concernés d'attester une formation continue séparée pour toutes les positions de droits acquis jusqu'à fin 2006 [1]. Le médecin qui n'a pas remis l'attestation de formation continue auto-déclarée jusqu'à cette date, a perdu le droit de facturer les positions tarifaires concernées. Malgré tout, la banque de données sur la valeur intrinsèque compte aujourd'hui encore 17000 médecins facturant un total d'1,2 million de positions de droits acquis.

\section{Le diplôme de formation continue en bref}

- Parmi les 45 programmes de formation continue, choisissez celui qui correspond à l'activité professionnelle principale exercée actuellement.

- Accomplissez aussi la formation continue pour les prestations qui ne sont pas couvertes par les titres en votre possession et qui se trouvent ainsi dans les droits acquis. Vous pouvez faire valoir ces formations continues hors discipline dans le cadre de la «formation continue élargie» (cf. graphique).

- Lorsque vous avez rempli toutes les conditions du programme de formation continue, demandez le diplôme de formation continue à la société de discipline médicale concernée.

\section{Instrument inefficace}

Compte tenu des expériences faites ces dernières années, la Commission paritaire de la valeur intrinsèque (PaKoDig) chargée de surveiller la banque de données est parvenue à la conclusion que la formation continue pour les prestations de droits acquis auto-déclarée n'était pratiquement pas contrôlable. Or il n'existe pas de standard permettant d'évaluer si une formation continue concrète est suffisante pour une position de droits acquis déterminée ou non. Sans possibilité de sanction, la formation continue stipulée pour les droits acquis devient un instrument inefficace, chronophage et générant des coûts très élevés, aussi bien pour les médecins concernés que pour la FMH qui gère la banque de données sur la valeur intrinsèque. C'est pourquoi, la question principale était la suivante: comment éviter que les 17000 médecins concernés doivent à nouveau remettre fin 2009 une attestation de formation continue pour conserver leurs positions de droits acquis dans la banque de données sur la valeur intrinsèque?

\section{Solution simple et pleinement efficace}

Après des négociations approfondies, la FMH et les répondants des coûts se sont mis d'accord pour une recette extrêmement simple et efficace: le diplôme de

- Le diplôme de formation continue vous servira de preuve pour la formation continue stipulée par la loi et celle exigée par le concept de la valeur intrinsèque pour les droits acquis.

- Le diplôme de formation continue doit être renouvelé tous les trois ans.

- Si vous ne disposez pas d'un diplôme de formation continue valable, vous devez continuer à déclarer la formation continue pour les prestations de droits acquis, position par position, par le biais de myFMH.

- Vous trouverez les 45 programmes de formation continue, les interlocuteurs des sociétés de discipline médicale et de plus amples informations sur www.siwf.ch $\rightarrow$ formation continue. 
formation continue. Désormais, la formation continue pour les positions de droits acquis est accomplie dans le cadre de la formation continue stipulée par la loi et attestée avec le diplôme de formation continue habituel [2]. Cette solution est devenue possible suite à la révision de la Réglementation pour la formation continue (RFC) qui a eu lieu l'année dernière et qui a introduit les nouveautés principales suivantes [3]:

- Désormais il n'est plus nécessaire d'accomplir, pour chaque titre acquis, le programme de formation continue correspondant. Il suffit de remplir le programme de formation continue qui correspond le plus à l'activité professionnelle exercée actuellement. Par exemple, un médecin exerçant princi-

\section{Questions fréquemment posées (FAQ)}

Vais-je perdre mon titre de spécialiste si je n'accomplis pas une formation continue? Non. La formation continue est un devoir professionnel dont le non-respect peut être sanctionné par les autorités sanitaires cantonales par un blâme ou une amende pouvant aller jusqu'à Fr. 20000.Le retrait du titre de spécialiste n'est pas possible.

Qui a l'obligation d'accomplir la formation continue?

Conformément à l'art. 9 de la Réglementation pour la formation continue (RFC), tous les détenteurs d'un titre postgrade fédéral ou d'un titre postgrade étranger reconnu (aussi celui de «Médecin praticien») sont tenus d'accomplir leur formation continue, aussi longtemps qu'ils exercent une activité médicale en Suisse. Les médecins qui se trouvent en formation postgraduée en vue d'un titre de spécialiste ne sont pas soumis à cette exigence. Cette disposition est également valable pour les personnes en formation qui possèdent déjà un titre de formation postgraduée.

J'ai acquis le titre de spécialiste en médecine interne et je me rends aux Etats-Unis pour travailler deux ans dans la recherche. Suis-je tenu d'accomplir une formation continue?

Non. Le devoir de formation continue s'applique uniquement aux médecins exerçant une activité médicale en Suisse (art. 9 RFC). II ne reprendra vigueur qu'à votre retour en Suisse. Cependant, comme la période de contrôle s'étend sur trois ans, il faut préciser que les séjours de courte durée à l'étranger (inférieurs à un an) ne suffisent pas pour suspendre le devoir de formation continue.

2 Vereinfachte Fortbildung für Besitzstandpositionen: Factsheet von Tarmedsuisse vom 23. Juni 2009 auf www.siwf.ch $\rightarrow$ Fortbildung $\rightarrow$ Newsletter und Infos (uniquement en allemand).

3 Hänggeli C, Giger M. La formation continue sans peine. Bulletin des médecins suisses. 2008;89(17):739-743. SUVA en tant que médecin porteur d'un titre de spécialiste en médecine interne. Dois-je accomplir une formation continue?

Le devoir de formation continue concerne tous les médecins qui exercent une activité médicale. Une telle activité existe lorsque vous examinez, conseillez ou soignez des patients sous une forme quelconque. Une activité administrative ou de re- palement dans le domaine cardiologique et titulaire des titres de spécialiste en «médecine interne», en «cardiologie» et en «médecine intensive» doit opter pour le diplôme de formation continue en cardiologie.

- Les 45 sociétés de discipline médicale définissent une «formation continue essentielle» de 25 heures par année. Les médecins peuvent effectuer les 25 autres heures en tant que «formation continue élargie» dans d'autres disciplines. Dans ce contexte, ils ont la possibilité de suivre une formation continue dans des domaines autres que ceux concernés par le titre de spécialiste acquis (prestations de droits acquis; cf. figure à la page suivante).

cherche sans contact avec des patients n'entre pas dans cette catégorie.

Que se passe-t-il si je n'acquiers pas un diplôme de formation continue?

L'acquisition du diplôme de formation continue n'est pas une obligation stipulée par la loi. Le titre de spécialiste reste intact. Le fait de ne pas posséder de diplôme de formation continue entraîne toutefois les inconvénients suivants:

- En cas de contrôle, vous devez convaincre les autorités cantonales concernées au moyen de documents appropriés que la formation continue que vous avez accomplie correspond au standard habituel. Il en va de même lors d'un procès éventuel de responsabilité civile.

- Sans l'inscription d'un diplôme de formation continue ou d'une attestation de formation continue dans le registre doctorfmh.ch, les assureurs peuvent refuser la prise en charge des positions de droits acquis. Le cas échéant, vous devriez confirmer la formation continue spéciale pour les prestations de droits acquis dans la banque de données sur la valeur intrinsèque et l'attester au moyen de documents adéquats [1].

Quel est le coût d'un diplôme de formation continue?

La reconnaissance de sessions de formation continue et le contrôle de la formation continue sont effectués par la société de discipline médicale concernée, laquelle perçoit des taxes en fonction des dépenses occasionnées. Pour les membres d'une société de discipline médicale, cette taxe est incluse généralement dans la cotisation annuelle. Pour les non-membres le diplôme valable pour trois ans ne devrait pas coûter plus de 150 francs. Des taxes plus élevées devront être approuvées par le Comité de I'ISFM si les coûts augmentent.
Le plénum de l'Institut suisse pour la formation médicale postgraduée et continue (ISFM) a approuvé ces «FAQ» le 26 novembre 2009 au sens de l'art. 13, $1^{\text {er }}$ al. de la Réglementation pour la formation continue (RFC). 


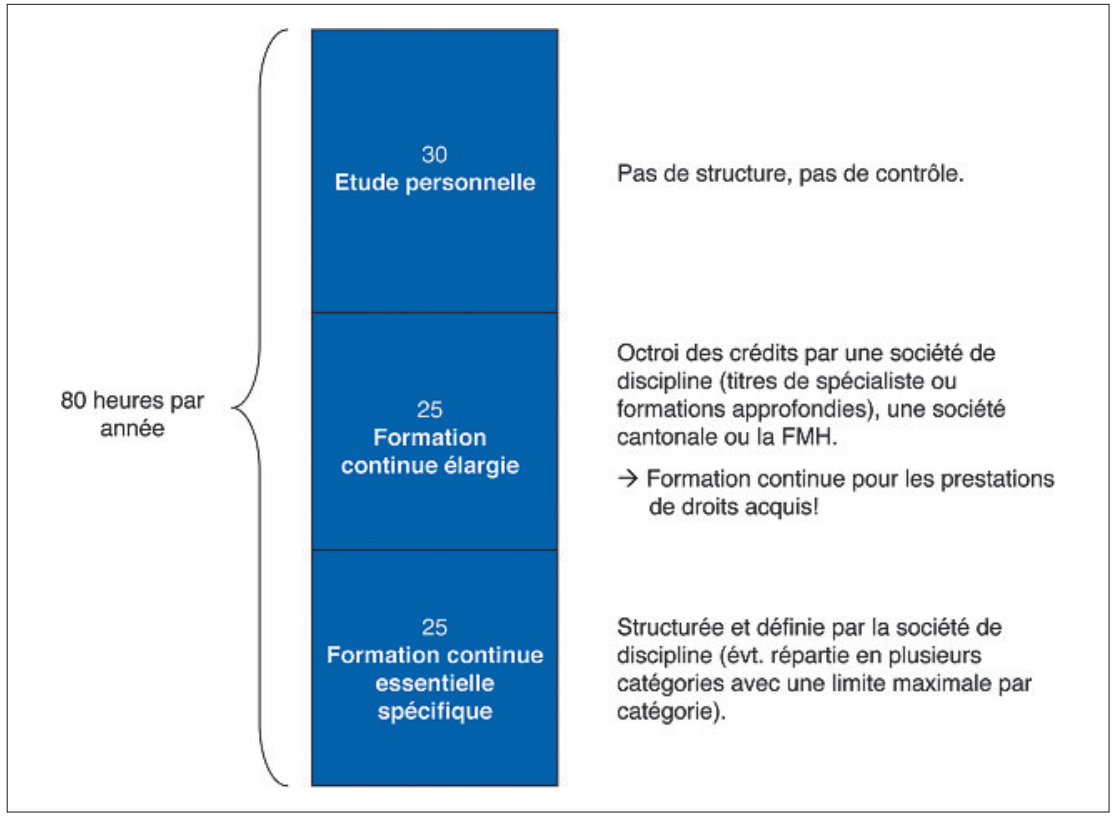

Structure des 80 heures de formation continue exigées par année.

\section{Deux avantages}

En acquérant le diplôme de formation continue, les médecins tenus de suivre une formation continue réalisent un double gain:

- Le diplôme de formation continue atteste que son titulaire a accompli le devoir de formation continue prescrit par la loi sur les professions médicales (LPMéd). Depuis le $1^{\text {er }}$ septembre 2007, les directions sanitaires cantonales peuvent sanctionner toute violation de ce devoir professionnel au moyen d'un blâme ou d'une amende jusqu'à 20000 francs.

- Le diplôme de formation continue vaut comme solution de rechange en lieu et place de la formation continue exigée jusqu'à présent pour les différentes positions de droits acquis.

Peu importe le programme choisi parmi les 45 programmes de formation continue, les médecins ont la possibilité de suivre une formation continue de 25 heures au plus par année dans d'autres disciplines selon leurs préférences et leurs besoins. Les diplômes de formation continue remis conjointement par les sociétés de discipline médicale et la FMH sont une offre de service exclusive pour leurs membres. Les non-membres obtiennent une attestation analogue servant aussi bien d'attestation pour le devoir de formation continue prescrit par la loi que pour la formation continue exigée pour les droits acquis. Et ce diplôme facilite accessoirement aussi le contrôle des assureurs qui peuvent savoir, en consultant le registre des médecins doctorfmh.ch, si les médecins remplissent leur devoir de formation continue ou non, ce qui les préserve de demandes inutiles.

\section{Perspectives}

Le devoir de formation continue prescrit par la loi et la possibilité d'attester la formation continue pour les positions de droits acquis avec le diplôme de formation continue conduiront le diplôme de formation à se développer en un standard général pour le droit de facturer à la charge de l'assurance-maladie. L'Institut suisse pour la formation médicale postgraduée et continue (ISFM) a décidé de créer une plate-forme de la formation continue. Cet instrument basé sur l'internet a pour but de contribuer à améliorer la qualité de la formation continue dès 2010 et d'alléger les travaux administratifs pour tous les médecins de même que pour les sociétés de discipline médicale et les sociétés cantonales de médecine.

En outre, les partenaires tarifaires ont l'intention de réviser le concept de la valeur intrinsèque; les exigences de formation postgraduée et continue ne devraient plus être définies au hasard, mais en fonction de l'objectif; de surcroit, elles devraient garantir la qualité des prestations fournies. Dans ce contexte, il convient de veiller à une réglementation libérale mettant l'accent sur la responsabilité et le libre exercice de la profession pour les médecins indépendants. Les prescriptions limitatives ne doivent pas seulement servir à assurer la qualité mais aussi être faciles à mettre en œuvre et à contrôler. Le nouveau concept de la valeur intrinsèque ne verra pas le jour avant l'été 2010. Nous vous tiendrons au courant.

\begin{tabular}{ll}
$\begin{array}{l}\text { Glossaire } \\
\text { Positions/prestations } \\
\text { tarifaires relevant } \\
\text { des droits acquis }\end{array}$ & $\begin{array}{l}\text { Tout médecin qui, avant le 1er janvier 2004, a fourni régulièrement et sans contestation } \\
\text { pendant trois ans des prestations peut les inscrire dans son catalogue des droits acquis et } \\
\text { continuer à les facturer même s'il n'est pas détenteur du titre correspondant. }\end{array}$ \\
$\begin{array}{l}\text { Valeur intrinsèque } \\
\text { qualitative }\end{array}$ & $\begin{array}{l}\text { La valeur intrinsèque qualitative indique quelles qualifications professionnelles (titre de spécialiste, } \\
\text { formation approfondie, attestation de formation complémentaire) sont nécessaires, en vertu } \\
\text { de la Réglementation pour la formation postgraduée, pour facturer des prestations spécifiques } \\
\text { à la charge de l'assurance sociale. La valeur intrinsèque qualitative de chaque prestation figure } \\
\text { dans le TARMED. }\end{array}$ \\
$\begin{array}{l}\text { Valeur intrinsèque } \\
\text { qualitative }\end{array}$ & $\begin{array}{l}\text { La banque de données sur la valeur intrinsèque permet à la FMH de gérer l'ensemble des données } \\
\text { fournies par les médecins lors du recensement des valeurs intrinsèques (positions de droits acquis, } \\
\text { attestations de formation continue). }\end{array}$ \\
$\begin{array}{l}\text { Concept de valeur } \\
\text { intrinsèque (version 9.0) }\end{array}$ & $\begin{array}{l}\text { Le concept de valeur intrinsèque } 9.0 \text { comprend toutes les réglementations passées entre } \\
\text { fournisseurs de prestations et assureurs en matière de droit à la facturation des prestations } \\
\text { conformément à la nouvelle structure tarifaire TARMED. }\end{array}$ \\
$\begin{array}{l}\text { Réglementation pour la } \\
\text { formation continue (RFC) }\end{array}$ & $\begin{array}{l}\text { La Réglementation pour la formation continue de la FMH règle le domaine d'application } \\
\text { et les principes de base de la formation continue médicale en Suisse. }\end{array}$ \\
\hline $\begin{array}{l}\text { PakoDig } \\
\text { La commission paritaire composée de représentants du corps médical et des assureurs est } \\
\text { chargée, entre autres, de surveiller et de contrôler la banque de données sur la valeur intrinsèque. }\end{array}$
\end{tabular}

